A RESPONSABILIDAD SOCIAL: del capitalismo salvaje al capitalismo benévolo

\title{
Riccardo Riccardi
}

Abogado. Doctor en Jurisprudencia - Universidad de Roma [Italia];

Licenciado de Honor en Ciencias Económicas - Universidad Comercial de Deusto-Bilbao [España];

Asesor en Planificación Estratégica del Rectorado de la Universidad Católica de Salta [Argentina];

Asesor de la Presidencia - Federación de Agrupaciones Santo Tomás de Aquino (FASTA),

Agrupación Internacional de Derecho Pontificio;

Conferencista internacional.

mriccardi@fibertel.com.ar

\section{Resumo}

El mundo generado por la globalización se caracteriza por la aparición de la responsabilidad social generalizada y extendida a todos los campos de la actividad humana como consecuencia de la diversidad y la complejidad que definen claramente las características del Tercer Milenio. En este nuevo contexto, la responsabilidad social de la Universidad es fundamental para que el tránsito del capitalismo salvaje al capitalismo benévolo se realice, no solamente acompañando a las nuevas tecnologías, sino que éstas tengan como marco la solidaridad y los principios éticos.

\section{Palabras clave}

Calidad de vida. Capitalismo benévolo. Globalización. Inteligencia social.

Responsabilidad social. 


\title{
Social responsibility: from wild capitalism to benevolent capitalism
}

\begin{abstract}
The world born of the globalization is marked by the emergence of social responsibility generalized and extended to all human activity fields as a consequence of the diversity and complexity that clearly define Third Milennium's aspects. In this new context, the University's social responsibility is fundamental to the actual passage from wild capitalism to benevolent capitalism, accompanied not only by new technologies but also by solidarity and ethical principles as a distinguishable mark.
\end{abstract}

\section{Key words}

Benevolent capitalism. Globalization. Life quality. Social intelligency.

Social responsability. 


\section{Introducción}

El siglo XXI ha confirmado, con abundancia de ejemplos, que la globalización de los tiempos modernos presenta características inéditas y que su materialización ha hecho pasar a un plano secundario la pregunta acerca de "[...] cuál es la verdadera condición del hombre [...]" en el nuevo entorno mundial y en este particular momento de la civilización.

Si partimos de unas tentativas de definición de la globalización, encontramos las siguientes acotaciones que pueden ser útiles si queremos contar con una panorámica que proporcione ángulos visuales diferenciados, cuyo conocimiento resultará indispensable para comprender mejor el mundo en que vivimos: "Todos invasores, todos invadidos." (RICCARDI, 2002, p. 214).

Esta síntesis, traducida en la práctica, indica:

a) que existe una intención de hacer caer las barreras comerciales entre todos los países;

b) que todos pueden exportar (si son capaces de hacerlo);

c) que todos deben aceptar ser mercado para los otros;

d) que en esta confrontación habrá siempre muertos y heridos;

e) que la apertura de los países a las importaciones no siempre es justa ni democrática.

También Luigi Zampetti (Globalización y culturas locales) advierte que:

La globalización - como fenómeno - se encuadra en el modelo de desarrollo que supone, tanto el proceso de mundialización, como el proceso de globalización que es subsecuente del primero.
El desarrollo en la sociedad moderna es entendido únicamente como un desarrollo de carácter económico y que, consecuentemente, no coincide con el concepto de desarrollo pleno y con el desarrollo humano en la unidad de sus dimensiones. (ZAMPETTI, 2002, p. 37).

Otro estudioso de política económica señala que:

La globalización no es un fenómeno nuevo; pero su proceso arrollador presenta aspectos desconocidos. El constante y vertiginoso impacto del factor tecnológico, el nuevo rol prevaleciente del conocimiento, la presencia imprescindible de los medios de comunicación que dotan al hombre del preciado don de la ubicuidad, de la simultaneidad y de la versatilidad de la información que son requisitos para la comprensión de la realidad que emerge a touts les azimuts (todas las direcciones) Estos son los hechos que debemos enfrentar reflexiva y prudentemente. (MuruÁ, 2002, p. 98).

La incapacidad de dar una respuesta al interrogante que hemos formulado ha sido reemplazada por toda una serie de propuestas - escasamente llevadas a la práctica - que enuncian una tentativa evidente de trasladar la cuestión a un plano diferente que permita más bien enmascarar la falta de una actitud de auténtica autocrítica, utilizando declaraciones altisonantes con las cuales se logra una duplicación del resultado que consiste en:

a) 'amauterizar' la verdadera 'condición humana' en la era de la globalización;

b) exaltar y magnificar 'las intenciones de mejorarla teóricamente', anunciando que ha llegado la era de la Responsabilidad Social. 
Nuestro propósito consiste en analizar:

- La transformación del capitalismo por el impacto de la globalización;

- La definición académica de la responsabilidad;

- La responsabilidad social (latu sensu);

- La responsabilidad social en las Instituciones de Enseñanza Superior (IES);

- La responsabilidad social y su impacto sobre la imagen corporativa;

- La responsabilidad social se materializa con el aporte de inteligencia social;

- La responsabilidad social como resultado.

\section{Del capitalismo salvaje al capitalismo benévolo}

Aunque parezca poco creíble, fue la Revolución Industrial del siglo XIX la primera que centró la atención sobre la Responsabilidad Social empresaria.

Esta sociedad constituía, parcialmente, un reflejo del nuevo poder que le era reconocido por los nuevos procesos industriales (lo que hoy llamamos tecnología), que hacían posible cambiar las relaciones del antiguo régimen, introduciendo cambios profundos en las relaciones entre individuos y grupos de la sociedad.

Jean-Jacques Rousseau, en su Discurso sobre el origen de la desigualdad entre los hombres, publicado en 1762 , ya advertía que la concentración del poder en las manos de la nueva clase de propietarios, levantaría sospechas respecto de su poder, de sus motivaciones y de sus métodos, y que terminarían proporcionando 'nuevos grilletes a los pobres y nuevos poderes a los ricos'.
Todavía en el siglo XX, algunos de sus más afamados estudiosos, como Friedman, Hayek, Ansoff y Heilbronner, han defendido la tesis que los negocios deben limitarse a los menesteres comerciales y esgrimen varias justificaciones para ello.

Friedman (1962, p. 16) en su tesis expresada en Capitalism and freedom propone el siguiente interrogante:

Si los hombres de negocios han de tener una responsabilidad distinta a la de realizar los máximos beneficios para sus accionistas, ¿cómo la reconocerán? ¿Pueden individuos escogidos por si mismos decidir cuál es el interés social? Existe una sola Responsabilidad Social de los negocios: utilizar sus recursos y emprender las actividades encaminadas al aumento de los beneficios, en tanto que respete las reglas del juego y entre en franca y libre competencia, sin engaño ni fraude.

Como se puede apreciar, la palabra 'hombre' brilla por su ausencia, lo que nuevamente se repite en la declaración de principios de la Task force on corporate social performance (DEPARTMENT of Commerce, 1980).

Las corporaciones mercantiles existen, en principio, para producir mercancías que la sociedad desea y necesita. El cumplimiento de este objetivo es su primera y principal responsabilidad. Si fracasa en esta misión, no puede esperar que asuma otras.

En el final del siglo XX y en los primeros momentos del siglo XXI, se ha podido observar que el capitalismo salvaje se ha dado cuenta que resulta prácticamente imposible persistir en una visión restringida de la responsabilidad empresarial y que hay que reconocer la necesidad de incorporar normas y valores que permitan pasar de la visión limitada propuesta por los teóricos del capitalismo 
salvaje, a otra visión más 'aceptable' a la que se puede acceder proponiendo una nueva denominación del capitalismo: el capitalismo benévolo.

Este tipo de capitalismo se identifica con la aparición de la solidaridad, considerada como una virtud que abre el camino intermedio entre el paternalismo y el afán de ganancia por un lado y, por el otro, la sed de poder a cualquier precio.

El capitalismo benévolo en el siglo XXI se encuentra en la obligación de ser protagonizado por empresas e instituciones interesadas en ser vistas como 'buenas ciudadanas'. Los mega escándalos proporcionados en estos primeros años del siglo XXI por empresas de gran porte no solo en los Estados Unidos (EEUU), sino también en Europa, han obligado al mundo empresarial a dedicarse seriamente a la ética y a la función social que hoy son valoradas por todos los actores (accionistas, cliente, personal y comunidad).

En síntesis, es posible afirmar que, partiendo de una visión limitada de la empresa, insensible a las normas éticas y a los valores, (como se observó desde el comienzo de la Revolución Industrial hasta 1980), nos estamos lentamente dirigiendo hacia un nuevo tipo de empresa superadora de las limitaciones propias del capitalismo salvaje y orientada a la incorporación firme de normas y valores éticos que permitan clasificarla como empresas con responsabilidad social.

A este punto, conveniente explicitar el contenido de la responsabilidad y, una vez que la hayamos definido, proceder a analizarla en su interpretación social.

\section{La definición académica de la responsabilidad}

Los tratados de filosofía se han ocupado seriamente de la responsabilidad y estas contribuciones invalorables facilitan nuestra búsqueda de la mejor definición académica de la responsabilidad.
Conviene anticipar que el concepto y el término responsabilidad, son de data relativamente reciente y aparecen por primera vez en inglés y en francés en el año 1787 en la obra de Hamilton (1943).

Los tratadistas que se han ocupado de la responsabilidad han llegado a la clasificación siguiente:

a) responsabilidad civil;

b) responsabilidad penal;

c) responsabilidad moral en una interpretación doble que la específica como:

c.1) obligación moral;

c.2) situación de un agente consciente de sus actos.

Debemos señalar que en los tratadistas consultados no se encuentra una referencia específica a la Responsabilidad Social. Esto nos permitiría aceptar una primera aproximación que la define como: situación o carácter de aquel que puede ser llamado a responder de un determinado hecho y también tener en cuenta una definición que la cataloga como: posibilidad de prever los efectos del comportamiento mismo a partir de tal previsión.

Partiendo del supuesto que estas dos definiciones de la responsabilidad (sin adjetivos) se complementan entre sí, estaríamos incluyendo en la definición primera el concepto de imputabilidad y en la segunda admitiríamos una cierta cuota de libertad, que es la que alimenta el comúnmente denominado sentido de responsabilidad.

\subsection{La Responsabilidad Social (latu sensu)}

Nos oponemos firmemente a la tesis que nos propone limitar la Responsabilidad Social a la empresa, y por esta razón abogamos por una Responsabilidad Social (latu sensu) que debe ser vivenciada por toda la sociedad sin distinciones. 
Cada uno aportará lo que debe aportar a esta responsabilidad con la sociedad y nadie podrá estar excusado por sus incumplimientos.

Solo sensibilizando nuestras sociedades acerca de la necesidad de cambio que impone la globalización, será posible imaginar una vida mejor para todos los participantes de la vida social.

Es evidente que la Responsabilidad Social será tarea preeminente en las sociedades democráticas del futuro inmediato, delante de problemas gigantescos planteados con dureza y hasta con violencia por la salud, la educación, la vivienda, la alimentación y la seguridad.

En estas áreas, la Responsabilidad Social inevitablemente será afrontada con la colaboración de todos los estamentos sociales Sería ilógico pensar que las empresas, aunque se consideran como la espina dorsal de la economía y el principal propulsor del desarrollo económico, sean sus únicas protagonistas, pero es innegable su aporte tecnológico y sus constantes innovaciones y por ello constituyen una fuerza esencial en el cambio.

Se puede, consecuentemente, hablar de una Responsabilidad Social colectiva, que será tanto más eficaz cuanto más esté relacionada con acciones colectivas provenientes de los distintos sectores de la sociedad.

Un gran aliado en el proceso de Responsabilidad Social en las décadas de 1960 y 1970, está representado por el crecimiento expansivo de las Organizaciones No Gubernamentales (ONG), que han superado las dimensiones del cooperativismo aprovechando la ductilidad de sus estructuras y el énfasis voluntarístico desestructurado que han sabido exhibir con gran éxito.

En este ensayo concentramos nuestro interés sobre las IES, que consideramos como el alimentador natural del proceso de Responsabilización Social, no solo por el prestigio que les deriva de su función educativa, sino por la posibilidad de exhibir una actitud crítica frente a la sociedad, difícil de encontrar en otros sectores.

En nuestra óptica, las instituciones - en general-alcanzan el nivel de Responsabilidad Social cuando satisfacen los imperativos siguientes: a) son capaces de incorporar sistemas de ética y de valores sociales en su contexto;

b) son capaces de plantearse las cuestiones que requieren cumplimiento;

c) son capaces de sostener con autoridad, firmeza y continuidad, sus compromisos con la Responsabilidad Social;

d) son capaces de satisfacer las necesidades de los más débiles (económica y socialmente);

e) son capaces de cumplir las responsabilidades que les exige el ambiente;

f) son capaces de conducir con firmeza los procesos de cambio impuestos por la globalización, realizándolo con un enfoque humano altamente perceptible;

g) son capaces de imponerse autocontroles severos y de conocimiento público, y actitudes transparentes, respetando el viejo concepto de: 'el pueblo quiere saber';

h) son capaces de proponer en todas sus acciones, objetivos desafiantes y de luchar incansablemente para lograrlos.

La Responsabilidad Social implica aceptar las siguientes realidades:

a) no hay evolución sin cambios. Se necesita innovar en las ideas y en los estilos de conducción. Hay que asumir nuevas actitudes y confiar en nuevas percepciones. Hay que imaginar nuevas estructuras y adoptar nuevos enfoques y hay que introducir los nuevos valores requeridos por el entorno y el momento;

b) los procesos que generan crecimiento económico (sostenido y sustentable [sostenible]), si no contienen implicaciones de 
valor son, - inevitablemente - incapaces de detectar las crisis subyacentes. No permiten un juicio correcto de valor porque las ideologías que acompañan a estas técnicas no se lo permiten;

c) en un mundo dominado por el marketing, la tentación de canalizar las acciones de la institución en clave (encubierta) de publicidad, propaganda, promoción, debe ser constantemente controlada para evitar que la Responsabilidad Social sea confundida con la beneficencia (verbal o escrita) vacía de contenido;

d) finalmente, la adopción plena de la Responsabilidad Social por parte de las empresas debe revelar claramente que ellas la aceptan haciéndose cargo de su materialización, reconociendo que tienen una obligación moral respecto de alguna cosa o por alguna persona;

e) en la actualidad, los mejores resultados obtenidos por la Responsabilidad Social se manifiestan en la comunidad, y vale la pena señalar que los objetivos deseados en materia de Responsabilidad Social pueden ser cabalmente logrados siempre y cuando las acciones que ella propone sean planificadas como cualquier otra actividad.

\section{2. la Responsabilidad Social y las IES}

La Universidad tiene hoy un compromiso social de grandes dimensiones que, además, tiende a acrecentarse continuamente. Por lo tanto, ella debe ampliar su acción preparando y desarrollando programas que habiliten a los alumnos para que éstos puedan enfrentar, con probabilidades de éxito, con seriedad y compromiso, los desafíos que les proponen las instituciones en las que trabajarán en el futuro.
A este punto surge legítima la pregunta: ¿Cómo puede contribuir la Universidad para que los diversos actores sociales expresen y ejerciten su Responsabilidad Social?

La respuesta puede ser resumida en las siguientes acotaciones: La Universidad logra su compromiso utilizando distintos caminos, que pueden ser recorridos conjunta o separadamente:

a) promoviendo las iniciativas sociales de los estudiantes que aprenden a trasladar inteligentemente a la realidad social lo que están aprendiendo en los cursos;

b) proponiendouna visión de la Responsabilidad Social Corporativa, compatible con el momento y el entorno en los cuales las instituciones se mueven, a los alumnos destinados a desempeñarse en un mundo complejo, globalizado y diversificado;

c) acompañando el fortalecimiento de las organizaciones sociales que pueden transformarse en 'una arena' en la que se exhibe un ejercicio colectivo y mancomunado de Responsabilidad Social;

d) contribuyendo a involucrar personas con recursos y vocaciones para emprender con 'óptica social', emprendimientos sociales que puedan generar un mundo más efectivo;

e) diseñando las actividades formativas, orientándolas para que puedan suministrar herramientas para que los alumnos formulen y realicen iniciativas que aporten beneficios a la comunidad o que contribuyan a mejorar las condiciones de vida de los sectores más débiles de la sociedad;

f) acercando sus alumnos a las organizaciones (tipo ONG) y a los emprendimientos generadores de soluciones audaces y modernas 
que permitan salir de la teoría abstracta para resolver en la práctica problemas comunitarios concretos;

g) aceptando la tesis de la indispensabilidad de la formación práctica de la solución de problemas reales, se descubre la conveniencia de utilizarlos como materia de divulgación y de estudio académico.

Además, los problemas reales constituyen una excelente ocasión para desarrollar trabajos de cambio de naturaleza compleja, así como investigaciones puntuales $\mathrm{o}$, simplemente, pueden ser utilizados como experiencia válida de aprendizaje de servicios.

a) precisando las implicancias y obligaciones morales que tal creación implica, señalando aquellas instituciones que no dan indicio de entender que el mundo cambia constantemente;

b) aceptando que la Universidad, para poder realmente cumplir su misión, debe ser capaz de capacitar a todas las personas que ejercen en su seno cargos directivos, ejecutivos y operativos en las áreas de Relaciones Institucionales y de Relaciones con la Comunidad y en las Fundaciones Corporativas que permiten a las empresas a canalizar su acción social de modo que este resulta más efectivo y eficiente;

c) contribuyendo, en su ámbito de generación de conocimiento, a mejorar las políticas y estrategias y prácticas de la Responsabilidad Social empresaria, investigando sistemáticamente los modelos que son utilizados por las empresas y, consecuentemente, para los entornos en los que operan e indirectamente para toda la comunidad; d) aprovechando su naturaleza docente para promover la Responsabilidad Social empresaria en un público muy amplio, interclasista, la Universidad cumple con la misión para la cual se la considera no solo idónea, sino que está en condiciones inigualables para cumplir su cometido frente a la comunidad. Este reconocimiento, que ha sido constante a lo largo de los siglos, se ha hecho más firme y sostenido en el siglo XX y se puede, con lógica, afirmar que aumentará todavía mucho más frente a los nuevos desafíos que provienen de los procesos tecnológicos e innovativos.

\section{El impacto de la Responsabilidad Social sobre la imagen corporativa}

Desde hace algunos años, el capitalismo benévolo se ha preocupado seriamente por la imagen proyectada por las instituciones que lo representan en un universo en el que aparecen en primera líneas los aparatos estatales, regionales, provinciales y municipales y las empresas públicas.

Siguen después las empresas privadas que exhiben las mismas preocupaciones y luego aparecen otras instituciones (como p. ej. las universidades y los centros educativos) que han comenzado a tomar muy en serio la evaluación que la comunidad hace de su imagen corporativa.

No escapan a esta ley férrea ni las instituciones religiosas, ni los media, ni las ONGs, ni las fuerzas armadas ni las corporaciones profesionales.

Podemos afirmar conscientemente que todas las instituciones, sin distinción, se preocupan hoy por ofrecer a sus comunidades una imagen corporativa positiva.

Pero para poder comprender mejor este fenómeno, es necesario analizar cómo se ha venido formando la imagen corporativa en las instituciones que, a partir de 1950, acuñaron el eslogan "Hacer bien y hacerlo saber". Esta declaración de 'buenas intenciones' era evidentemente pobre para satisfacer los deseos (o las necesidades) de la 
sociedad y, además, se limitaba generalmente a una serie de acciones puntuales y ocasionales en las que se mezclaban la caridad, la misericordia, la beneficencia, el deseo de limpiar la conciencia frente a las críticas que proporcionaba el propio capitalismo salvaje.

La complejidad y la diversidad de las problemáticas relacionadas con el Factor Humano produce, a partir de 1960, un 'ablande' del capitalismo salvaje hacia una forma más aceptable que hemos definido como benévolo, más (o esencialmente) en la forma que en la sustancia.

A partir de fines del siglo XX y con particular énfasis en los primeros años del siglo XXI comienza a tomar consistencia el concepto de Responsabilidad Social.

A este punto nos parece conveniente intentar una primera definición de la imagen corporativa identificándola como 'el producto lógico de un proceso institucional que se origina primariamente en la cultura de la institución, cultura que, a su vez, genera una identidad corporativa que es única, en el sentido que cada institución tiene su cultura que es propia y exclusiva'.

Solamente cuando la empresa llega a identificar qué tipo de cultura representa, se podrá hablar de una identidad corporativa que realizándose en determinadas y específicas acciones obtiene como resultado una imagen corporativa 'única' del mismo modo que lo es la cultura.

En otros trabajos hemos explicitado que la imagen corporativa es la 'conclusión lógica de un proceso extenso' que viene puesta en marcha dentro de cada empresa de un modo original e irrepetible.

Es necesario tener siempre presente que la tríada cultura-identidad-imagen tienen tiempos de materialización distintos.

Las actividades que, partiendo de la cultura conducen a la definición de la identidad corporativa (que es a su vez el soporte conceptual de la imagen que se proyecta a la sociedad) son innumerables y cubren prácticamente todos los campos de la actividad humana.

La imagen corporativa se concreta en los resultados que las instituciones son capaces de producir.
Los sectores productores de imagen más activo se centran - en nuestra época - en los dominios de la educación, la salud, la alimentación y la vivienda, para limitarnos a los principales, porque hay toda una gama de actividades derivadas que es prácticamente imposible enumerar con exactitud.

El rasgo característico de la Responsabilidad Social se expresa en el énfasis que cada institución es capaz de poner en sus acciones, enfatizando siempre que lo que se hace sirve esencialmente para medir con exactitud la propia actitud proactiva dirigida al bien común.

\section{La responsabilidad social requiere inteligencia social}

La Responsabilidad Social para pasar a la acción necesita indispensablemente contar, en sus ejecutores, con un tipo de inteligencia particular: la Inteligencia Social.

Esta particular inteligencia, de reciente adquisición, aparece en los estudios más avanzados acerca de la conducta humana y cuenta con la contribución de un estudioso de reputación mundial: estamos hablando de Tony Buzán, creador de los Mapas Mentales, definidos por él como "[...] un método de análisis que permite utilizar al máximo las capacidades de la mente." (p. 147) Buzán, en su libro El poder la inteligencia social, enumera (en el capítulo titulado $E l$ poder del diez) las inteligencias que todos disponemos. Citamos las palabras del propio Buzán (2003, p. 147):

La Inteligencia Social es sólo una de las diez inteligencias que todos tenemos. La inteligencia se ha dividido tradicionalmente en tres aspectos diferentes: verbal, numérico y espacial. Sin embargo, también tenemos inteligencias que definimos como creativa, sensual, física, personal, sexual y espiritual. Lo maravilloso respecto a estas múltiples inteligencias es que cada una trabaja en conjunto con las demás y las refuerza de 
forma sinérgica. Cuando desarrollas una de tus inteligencias, simultáneamente las desarrollas a todas.

La definición de (id. ib., p. 27) de la Inteligencia Social dice: "La inteligencia social simplemente es cómo nos llevamos y cómo nos relacionamos con las personas que nos rodean."

No tenemos duda alguna: la Responsabilidad Social plenamente entendida, necesita contar con la Inteligencia Social.

\section{La Responsabilidad Social como resultado}

Como conclusión, queremos contribuir con nuestra definición de la Responsabilidad Social identificándola como una secuencia o proceso que, partiendo de la cultura corporativa, modela la identidad corporativa y confluye en la imagen corporativa.

Podemos también considerarla como el resultado de acciones que son evaluadas midiendo las opiniones de los destinatarios, de sus acciones y finalmente, podemos también clasificarla como una prueba de madurez de la institución, que así gana el derecho de ser clasificada como empresa socialmente responsable.

Adoptando estaúltima interpretación, observaremos que la empresa responsable debe exhibir, necesariamente, las siguientes características:

a) ejercer su función de gobierno con integridad;

b) desarrollar un trabajo comprometido y coherente con la misión que se ha propuesto cumplir;

c) vivenciar en su accionar diario los valores que ha anunciado adoptar;

d) aceptar mentalmente ser parte de un colectivo más amplio, que considera valores similares; e) integrar sus decisiones de modo coherente en el terreno económico, social y medioambiental;

f) rendir cuenta del resultado de su actuación a toda la sociedad;

g) separar mental y claramente otras actividades colaterales (publicidad, propaganda, publicidad) de su responsabilidad frente a la ética y a los valores, relegando estas actividades al papel de 'coadyuvantes' sin caer en la tentación de considerarlas algo más que herramientas o medios útiles para el logro del fin social.

Finalmente, se puede afirmar que este siglo asistirá a un crescendo de sensibilidad social abierta y declarada, que reclamará cuotas siempre más consistentes de Responsabilidad Social, necesaria para pensar de alcanzar un mundo, si no perfecto, por lo menos mucho menos imperfecto de lo que es hoy.

\section{Referencias}

BuzÁN, Tony. El poder la inteligencia social. Barcelona: Ediciones Urano, 2003.

Department of Commerce. Task force on corporate social performance. In: Business and society: strategies for 1980. Washington, DC: Department of Commerce, 1980.

Friedman. Capitalism and freedom. Chicago: University of Chicago Press, 1962.

Hamilton, Alexander. El federalista o la nueva constitución. México: Fondo de Cultura Económico, 1943.

MuruÁ, Patricio Colombo. La globalización no es un fenómeno nuevo. Primer Forum Mundial Nord-Sud. Roma: Franco Angeli Editore, 2002.

Riccardi, Riccardo. Primer Fórum Mundial Nord-Sud. Roma: Franco Angeli Editore, 2002.

ZAMPETTI, Luigi. Globalización y culturas locales. Primer Fórum Mundial Nord-Sud. Roma: Franco Angeli Editore, 2002. 